\title{
CONGRUENCE LATTICES OF FUNCTION LATTICES
}

\author{
G. GRÄTZER AND E. T. SCHMIDT
}

\begin{abstract}
The function lattice $L^{P}$ is the lattice of all isotone maps from a poset $P$ into a lattice $L$.

D. Duffus, B. Jónsson, and I. Rival proved in 1978 that for a finite poset $P$, the congruence lattice of $L^{P}$ is a direct power of the congruence lattice of $L$; the exponent is $|P|$.

This result fails for infinite $P$. However, utilizing a generalization of the $L^{P}$ construction, the $L[D]$ construction (the extension of $L$ by $D$, where $D$ is a bounded distributive lattice), the second author proved in 1979 that Con $L[D]$ is isomorphic to $(\operatorname{Con} L)[\operatorname{Con} D]$ for a finite lattice $L$.

In this paper we prove that the isomorphism $\operatorname{Con} L[D] \cong(\operatorname{Con} L)[\operatorname{Con} D]$ holds for a lattice $L$ and a bounded distributive lattice $D$ iff $\operatorname{Con} L$ or $D$ is finite.
\end{abstract}

\section{INTRODUCTION}

For a lattice $L$ and a poset $P$, let $L^{P}$ denote the set of all order-preserving maps of $P$ to $L$ partially ordered by $f \leq g$ if and only if $f(x) \leq g(x)$, for all $x \in P$. Then $L^{P}$ is a lattice; it is called a function lattice. This lattice plays a major role in lattice theory.

D. Duffus, B. Jónsson, and I. Rival [2] (see also [1]) obtained the following result:

Theorem 1. Let $L$ be a lattice, and let $P$ be a finite poset. If $P$ has $n$ elements, then the following isomorphism holds:

$$
\text { Con } L^{P} \cong(\operatorname{Con} L)^{n} \text {. }
$$

It is evident that Theorem 1 does not remain valid for an infinite poset $P$. However in [8], using Priestley's representation of distributive lattices, the second author generalized a special case of this result (namely, the case of a finite lattice $L)$, as follows.

Let $D$ be a bounded distributive lattice, and let $X$ denote the poset of all ultrafilters of $D$ with the usual topology. For a lattice (or in general, for a join-semilattice) $L$, let $L[D]$ denote the lattice (resp, join-semilattice) of all continuous isotone maps of the space $X$ into the discrete space $L$; we call $L[D]$ a generalized function (semi-) lattice. The constant maps form a sublattice of $L[D]$; we identify $L$ with this sublattice. It is easy to see that for a finite distributive lattice $D$, the lattice $L[D]$ is a function lattice, namely, $L[D]$ is isomorphic to $L^{P}$ where $P=J(D)$ is the poset of join-irreducible elements of $D$.

Date: April 2, 2000.

1991 Mathematics Subject Classification. Primary 06B10; Secondary 08A05.

Key words and phrases. Congruence lattice, function lattice.

The research of the first author was supported by the NSERC of Canada.

The research of the second author was supported by the Hungarian National Foundation for Scientific Research, under Grant No. 1903. 
The result from E. T. Schmidt [8] is as follows:

Theorem 2. Let $L$ be a finite lattice, and let $D$ be a bounded distributive lattice. Then

$$
\operatorname{Con} L[D] \cong(\operatorname{Con} L)[\operatorname{Con} D]
$$

Our main result in this paper is the following:

Theorem 3. Let $L$ be a lattice, and let $D$ be a bounded distributive lattice. Then the isomorphism

$$
\operatorname{Con} L[D] \cong(\operatorname{Con} L)[\operatorname{Con} D] .
$$

holds if and only if either Con $L$ is finite or $D$ is finite.

Corollary . Let $L$ be a lattice. If the isomorphism (Con) holds for an arbitrary bounded distributive lattice $D$, then $\operatorname{Con} L$ is finite.

And conversely, if $\operatorname{Con} L$ is finite, then the isomorphism (Con) holds for an arbitrary bounded distributive lattice $D$.

The statement in Theorem 3 that the isomorphism (Con) holds if $D$ is finite is just a restatement of Theorem 1. Indeed, if $D$ is finite, then $L[D]$ is isomorphic to $L^{P}$, where $P=J(D)$; and Con $D$ is the Boolean lattice $2^{n}$, so $(\operatorname{Con} L)^{n}$ is the same as $(\operatorname{Con} L)[\operatorname{Con} D]$.

The other positive statement in Theorem 3, namely, that the isomorphism holds if Con $L$ is finite, generalizes Theorem 2. Schmidt's pro of of Theorem 2 is based on the observation that $L[D]$ is an extension of $L$ in which every prime interval of $L$ contains a copy of $D$. So it is somewhat surprising that we can generalize Theorem 2 to non-discrete lattices.

Theorem 3 shows that these two positive results are best possible. If Con $L$ is infinite and $D$ is also infinite, then the isomorphism (Con) always fails.

In Section 2, we shall investigate congruence lattices of function lattices with finite exponent. We give a very short proof of Theorem 1, and we also prove some results needed in the proof of the main result. In Section 3, we shall show (Theorem 4) that by working with $\operatorname{Comp} L$, the join-semilattice of compact congruences rather than with Con $L$, we can generalize Theorem 2 from finite lattices to arbitrary lattices.

The main result is presented in Section 4. The proof of this result is based on the results of the previous sections and on Theorem 5 which investigates when the free distributive product of two algebraic distributive lattices is not complete.

\section{The CASE OF FINITE EXPONENT}

We start by giving a very brief proof of Theorem 1 .

Proof of Theorem 1. Let $L$ be a lattice, and let $P$ be a finite poset. $L^{P}$ is a subdirect product of $n$ copies of $L$; therefore, $L^{P}$ has congruences $\Phi_{1}, \ldots, \Phi_{n}$ such that the intersection of these congruences is $\omega$ and the quotient lattices are isomorphic to $L$. By congruence distributivity, $\operatorname{Con} L^{P}$ can be embedded into (Con $\left.L\right)^{n}$ (more generally, if $S$ is a subdirect product of the lattices $L_{1}, \ldots, L_{n}$, then Con $S$ is a sublattice of the direct product of $\left.\operatorname{Con} L_{1}, \ldots, \operatorname{Con} L_{n}\right)$. Therefore, to prove Theorem 1, it is sufficient to verify that distinct congruences of $L^{n}$ have distinct restrictions. 
So let $\Theta \neq \Phi$ be two distinct congruences of $L^{n}$; we represent them in the form $\Theta=\Pi\left(\Theta_{p} \mid p \in P\right)$ and $\Phi=\Pi\left(\Phi_{p} \mid p \in P\right)$, where $\Theta_{p}, \Phi_{p}, p \in P$, are congruences of $L$. Since $\Theta \neq \Phi$, there exists an $r \in P$ such that $\Theta_{r} \neq \Phi_{r}$. So we can choose $a<b \in L$ such that $a \equiv b\left(\Theta_{r}\right)$ but $a \equiv b\left(\Phi_{r}\right)$ fails (or symmetrically). Define a, $\mathbf{b} \in L^{n}$ as follows:

$$
\mathbf{a}(p)=\left\{\begin{array}{ll}
a, & \text { if } p \leq r ; \\
b, & \text { otherwise; }
\end{array} \quad \mathbf{b}(p)= \begin{cases}a, & \text { if } p<r ; \\
b, & \text { otherwise }\end{cases}\right.
$$

Then $\mathbf{a}, \mathbf{b} \in L^{P} ;$ moreover, $\mathbf{a} \equiv \mathbf{b}(\Theta)$ holds and $\mathbf{a} \equiv \mathbf{b}(\Phi)$ fails. This completes the proof of Theorem 1.

The lattice $L^{P}$ has the following interesting property. For $u \in L$ and $r \in P$, define the element $\mathbf{u}_{r} \in L^{P}$ as follows:

$$
\mathbf{u}_{r}(p)= \begin{cases}1, & \text { if } r<p \\ u, & \text { if } r=p \\ 0, & \text { ot herwise }\end{cases}
$$

For every $r \in P$, the sublattice $L_{r}=\left[0_{r}, 1_{r}\right]$ is a convex sublattice of $L^{n}$. It is easy to see that $L_{r}$ is a congruence class of the congruence relation $\Theta_{r}$ of $L^{n}$, yielding another proof of Theorem 1.

Let Comp $A$ denote the join-semilattice with zero of compact congruences of the algebra $A$. With this notation, we can state an easy consequence of Theorem 1:

Corollary . Let $L$ be a lattice, and let $P$ be a finite partially ordered set. If $P$ has $n$ elements, then the following isomorphism holds:

$$
\operatorname{Comp} L^{P} \cong(\operatorname{Comp} L)^{n} \text {. }
$$

Proof. Indeed, in the proof of Theorem 1 we verify that if $\Theta$ is a congruence relation of $L^{P}$, and we take the congruence $\bar{\Theta}$ of $L^{n}$ generated by $\Theta$, then $\bar{\Theta}$ restricted to $L^{P}$ yields $\Theta$. Now the Corollary follows since if $\Theta$ is compact, then so is $\bar{\Theta}$.

Another property for finite exponents, stated in terms of finite distributive extensions, is the following:

Lemma 1. Let $D_{1}$ be a $\{0,1\}$-sublattice of a finite distributive lattice $D_{2}$. Then $L\left[D_{2}\right]$ is an extension of $L\left[D_{1}\right]$, and the Congruence Extension Property holds.

Proof. Let $P_{i}$ denote the dual of the poset of join-irreducible elements $J\left(D_{i}\right)$ of $D_{i}$, $i=1,2$. The duality between finite distributive lattices and finite posets provides an isotone map $\varphi: P_{2} \rightarrow P_{1}$ (namely, $\varphi(x)$ is the smallest element in $D_{1}$ containing $x$, for $\left.x \in J\left(D_{2}\right)\right)$.

We define an embedding of $L^{P_{1}}$ into $L^{P_{2}}$. Let $f$ be an element of $L^{P_{1}}$. Then $f$ is an isotone map: $P_{1} \rightarrow L$; consequently, $\bar{f}=f \circ \varphi$ is an isotone map from $P_{2}$ into $L$, so $\bar{f} \in L^{P_{2}}$.

$L^{P_{1}}$ is a subdirect power of $L$; consequently, every congruence relation $\Theta$ of $L^{P_{1}}$ is a restriction of a congruence $\Pi\left(\Theta_{p} \mid p \in P_{1}\right)$ of $L^{\left|P_{1}\right|}$ to $L^{P_{1}}$.

Now we consider the congruence $\bar{\Theta}=\Pi\left(\Theta_{\varphi(q)} \mid q \in P_{2}\right)$ of $L^{\left|P_{2}\right|}$. It is easy to see that the restriction of $\bar{\Theta}$ to $L^{P_{2}}$ is an extension of the congruence $\Theta$ of $L^{P_{1}}$.

Finally, we state an interesting property of function lattices. This result was first proved for finite exponents as a crucial step in the proof of our main result. We state here for arbitrary exponents. 
Lemma 2. Let $K$ be a lattice, and let $P$ be a poset. Then

$$
\operatorname{Id} K^{P} \cong(\operatorname{Id} K)^{P} \text {. }
$$

Proof. For $F \in \operatorname{Id} K^{P}$, we define a map $\varphi^{F}$ of $P$ to $\operatorname{Id} K$ :

$$
\varphi^{F}: p \rightarrow p \varphi^{F}=\{p f \mid f \in F\} .
$$

$p \varphi^{F}$ is an ideal of $K$. Indeed, if $x, y \in p \varphi^{F}$, then $x=p f$ for some $f \in F$ and $y=p g$ for some $g \in F$; therefore, $x \vee y=p f \vee p g=p(f \vee g)$ and $f \vee g \in F$, since $F$ is an ideal of $K^{P}$. If $x \leq y \in p \varphi^{F}$, then $y=p f$ for some $f \in F$. Define $g \in K^{P}$ by $q g=q f \wedge x$, for $q \in p$. Then $g \leq f \in F$, so $f \in F$. Therefore, $x \in p \varphi^{F}$, proving that it is an ideal.

$\varphi^{F}$ is obviously an isotone map.

Now we can set up the map $\alpha$ from $\operatorname{Id} K^{P}$ to $(\operatorname{Id} K)^{P}$ :

$$
\alpha: F \rightarrow \varphi^{F} \in(\operatorname{Id} K)^{P}, \text { for } F \in \operatorname{Id} K^{P} \text {. }
$$

$\alpha$ is obviously an isotone map from $\operatorname{Id} K^{P}$ to $(\operatorname{Id} K)^{P}$. It is also one-to-one since $F \alpha$ determines $F$ :

$$
f \in F \quad \text { iff } \quad p f \in p \varphi^{F} \text {, for all } p \in P .
$$

We see that $\alpha$ is onto by describing its inverse, $\beta$ :

$$
\beta: \Phi \rightarrow F_{\Phi}=\left\{f \in K^{P} \mid p f \in p \Phi, \text { for all } p \in P\right\} .
$$

So $\alpha$ is an isomorphism between $\operatorname{Id} K^{P}$ and $(\operatorname{Id} K)^{P}$.

\section{Compact congruences}

The isomorphism in Theorem 2 does not hold in general. But we can prove a version of it in the general case by switching from congruence lattices to joinsemilattices of compact congruences.

Theorem 4. Let $L$ be a lattice, and let $D$ be a bounded distributive lattice. Then

$$
\operatorname{Comp} L[D] \cong(\operatorname{Comp} L)[\operatorname{Comp} D] \text {. }
$$

Let $B_{D}$ denote the Boolean lattice generated by $D$. Then Comp $D$ is isomorphic to $B_{D}$. So the isomorphism of the theorem can be restated as follows:

$$
\operatorname{Comp} L[D] \cong(\operatorname{Comp} L)\left[B_{D}\right] .
$$

Let $K$ be a join-semilattice with zero; let Id $K$ denote the ideal lattice of $K$. Theorem 4 has the following consequence:

Corollary 1 . Let $L$ be a lattice, and let $D$ be a bounded distributive lattice. Then the following isomorphism holds:

$$
\operatorname{Con} L[D] \cong \operatorname{Id}((\operatorname{Comp} L)[\operatorname{Comp} D]) \text {. }
$$

Corollary $\mathbf{2}$. Let $L$ be a lattice, and let $D$ be a bounded distributive lattice. Then the following isomorphism holds:

$$
\operatorname{Con} L[D] \cong \operatorname{Id}((\operatorname{Comp} L) *(\operatorname{Comp} D)) \text {. }
$$

In the last corollary, $*$ denotes the bounded free product of bounded distributive lattices.

We need three lemmas to prove Theorem 4 and its corollaries. The first is due to R. W. Quackenbush [7]: 
Lemma 3. Let $D_{1}$ and $D_{2}$ be bounded distributive lattices. Then

$$
D_{1}\left[D_{2}\right] \cong D_{1} * D_{2}
$$

In particular,

$$
D_{1}\left[D_{2}\right] \cong D_{2}\left[D_{1}\right]
$$

Consider the direct limit system of algebras

$$
\Re=\left\{A_{i}, \varphi_{i, j} \mid i, j \in I, i \leq j\right\} ;
$$

see, e.g., [3]. Thus $I$ is an updirected poset, $A_{i}, i \in I$ are algebras; $\varphi_{i, j}$ is a homomorphism of $A_{i}$ into $A_{j}$, for $i \leq j$. These are subject to the usual conditions.

An element $\mathbf{v}$ of the direct limit $\lim A_{i}$ is a vector such that $\mathbf{v}(i) \in A_{i}$, and for some $h \in I, \mathbf{v}(i)$ is defined for all $h \leq \vec{i}$; the components satisfy the usual condition: $\mathbf{v}(i) \varphi_{i, j}=\mathbf{v}(j)$ for $i \leq j$.

For $i \leq j(i, j \in I)$ and a congruence $\Phi_{i}$ of $A_{i}$, form the congruence $\Phi_{j}$ of $A_{j}$ generated by the $\varphi_{i, j}$ image of $\Phi_{i}$ in $A_{j}$. Obviously, if $\Phi_{i}$ is compact in $A_{i}$, then $\Phi_{j}$ is compact in $A_{j}$. So we obtain a direct limit system Comp $\Re$.

The following lemma is a generalization of Lemma 2.4 of M. Tischendorf [9].

Lemma 4.

$$
\lim _{\rightarrow} \operatorname{Comp} A_{i} \cong \text { Complim } \rightarrow A_{i} .
$$

Proof. If $\Phi$ is a compact congruence of $\lim \operatorname{Comp} A_{i}$, then

$$
\Phi=\Theta\left(\mathbf{a}^{1}, \mathbf{b}^{1}\right) \vee \ldots \vee \Theta\left(\mathbf{a}^{n}, \mathbf{b}^{n}\right) .
$$

Choose any $k \in I$ for which all these vectors are defined. Obviously,

$$
\Phi(k)=\Theta\left(\mathbf{a}^{1}(k), \mathbf{b}^{1}(k)\right) \vee \ldots \vee \Theta\left(\mathbf{a}^{n}(k), \mathbf{b}^{n}(k)\right)
$$

is a compact congruence of $A_{k}$. Mapping $\Phi$ to $\langle\Phi(i)\rangle$ gives the isomorphism of this lemma.

Let $\Re_{D}$ be the family of all finite $\{0,1\}$-sublattices of a bounded distributive lattice $D$, with set inclusion as the partial ordering and embeddings as maps. Obviously, $\lim \Re_{D} \cong D$.

For a bounded distributive lattice $E$, we can form $B_{E}$, the Boolean lattice generated by $E$. With the obvious definition, the Boolean lattices $B_{E}, E \in \Re_{D}$, form a direct limit system, with limit isomor phic to $B_{D}$.

Let $L$ be a lattice. For $E \in \Re_{D}$, we can form $L[E]$. The lattices $L[E]$ with the induced set inclusions and embeddings form a direct limit system $\Re_{D, L}$. Obviously, the direct limit of this system is $L[D]$.

Now we are ready to prove Theorem 4 .

Proof of Theorem 4. Let $L$ and $D$ be given as in the theorem. We form the direct limit system $\Re_{D, L}$. By the Corollary to Theorem 1 , for any $E \in \Re_{D}$,

$$
\operatorname{Comp} L[E] \cong(\operatorname{Comp} L)\left[B_{E}\right]
$$

(where in the right-side of this equation we use the definition of a join-semilattice extended by a bounded distributive lattice).

By Lemma 4, taking the limit of the left-side of the last equation, we obtain

$$
\lim _{\rightarrow} \operatorname{Comp} L[E] \cong \operatorname{Comp} \lim \rightarrow L[E] \cong \operatorname{Comp} L[D] .
$$


Taking the limit of the right-side of this equation, we get

$$
\lim _{\rightarrow} \operatorname{Comp} L\left[B_{E}\right] \cong(\operatorname{Comp} L)\left[B_{D}\right],
$$

obtaining the isomorphism (Comp).

Taking the ideal lattice of both sides of the isomorphism (Comp), we get Corollary 1. By applying Lemma 3 to Corollary 1, we obtain Corollary 2.

\section{The MAIN ResulT}

To prove our main theorem, we need the following result:

S:main

T:brandnew

Theorem 5. Let $E$ be a complete distributive lattice with an infinite ascending chain. Let $A$ be a complete distributive algebraic lattice with an infinite completejoin independent antichain. Then $E[A]$ is not complete.

Proof. Let $d_{1}<d_{2}<\cdots<d_{n}<\ldots$ be an infinite ascending chain in $E$. Set $\delta=\bigvee\left(d_{i} \mid i<\omega\right)$. Let $\left\{a_{i} \mid 0<i<\omega\right\}$ be an infinite complete-join independent antichain in $A$. Set $\alpha=\bigvee\left(a_{i} \mid i<\omega\right)$.

Let $L=E[A]$. By Lemma $3, L$ is isomorphic to $E * A$, the free boundeddistributive product. We shall prove that the set

$$
X=\left\{d_{1} \wedge a_{1}, d_{2} \wedge a_{2}, \ldots, d_{n} \wedge a_{n}, \ldots\right\}
$$

has no join in $E * A$. First, we verify some easy claims.

Claim 1. Let $d \in E$ and $a \in A$. Then $d \vee a$ is an upper bound of $X$ iff $d_{i} \leq d$ or $a_{i} \leq a$, for every $i<\omega$.

Proof. By the structure theorem of free bounded-distributive products ([4]),

$$
d_{i} \wedge a_{i} \leq d \vee a \quad \text { iff } \quad d_{i} \leq d \text { or } a_{i} \leq a .
$$

For $d \in E$, we define the elements $\delta(d)$ and $\alpha(d)$ of $E$ as follows:

a) If $d_{i} \leq d$ for all $i<\omega$, then $\delta(d)=\delta$ and $\alpha(d)=0$.

b) If $d_{i} \leq d$ and $d_{i+1} \leq \leq d$, then $\delta(d)=d_{i}$ and $\alpha(d)=\bigvee\left(a_{j} \mid i<j<\omega\right)$.

c) If $d_{i} \not \leq d$ for all $i<\omega$, then $\delta(d)=0$ and $\alpha(d)=\alpha$.

Claim 2. $d \vee a$ is an upper bound of $X$ iff $\delta(d) \leq d$ and $\alpha(d) \leq a$.

Proof. This is obvious by the definition of $\delta(d)$ and $\alpha(d)$.

Claim 3. An element $d \vee a$ is minimal in the set

$$
\{d \vee a \mid d \vee a \text { is an upper bound of } X\}
$$

iff $\delta(d)=d$ and $\alpha(d)=a$.

Proof. This is obvious since if $a \vee d$ is an upper bound of $X$, then so is $\delta(d) \vee \alpha(d)$.

Let $\varepsilon_{0}=\alpha, \varepsilon_{\omega}=\delta$, and for every $0<i<\omega$, let $\varepsilon_{i}=\delta\left(d_{i}\right) \vee \alpha\left(d_{i}\right)$. With this notation, we restate the previous claim:

Claim 4. If $\delta<1$, then $\left\{\varepsilon_{0}, \varepsilon_{1}, \ldots, \varepsilon_{n}, \ldots, \varepsilon_{\omega}\right\}$, otherwise, $\left\{\varepsilon_{0}, \varepsilon_{1}, \ldots, \varepsilon_{n}, \ldots\right\}$ is the set of all minimal upper bounds of $X$ of the form $d \vee a$. 
Claim 5. Any upper bound $u$ of $X$ can be represented in the form $u=u_{1} \wedge \ldots \wedge u_{n}$, where $1 \leq n<\omega$, and every $u_{j}$ contains some $\varepsilon_{i_{j}}, 0 \leq i_{j} \leq \omega ; i_{j}=\omega$ is permitted if $\delta<1$.

Proof. Every element $u$ of $E * A$ can be represented as a finite meet of elements of the form $d \vee a$, where $d \in E$ and $a \in A$. If $u$ is an upper bound of $X$, then so are its components $d \vee a$, so this claim follows from the previous claim.

Now we are ready to prove the theorem. Let us assume that $u=\bigvee X$ exists. Then by the last claim, $u$ has a representation of the form $u=\varepsilon_{i_{1}} \wedge \varepsilon_{i_{2}} \wedge \ldots \wedge \varepsilon_{i_{n}}$, where $0 \leq i_{1}<i_{2}<\cdots<i_{n} \leq \omega ; i_{n}=\omega$ is permitted if $\delta<1$.

We shall distinguish four cases.

Case 1. $u=\alpha$. Then $u \wedge \varepsilon_{1}$ is an upper bound for $X$. Since $a_{1}<u$ but $a_{1} \not \leq u \wedge \varepsilon_{1}$, it follows that $u \wedge \varepsilon_{1}<u$, contradicting $u=\bigvee X$.

Case 2. $u=\delta<1$. Then $u \wedge \varepsilon_{1}$ is an upper bound for $X$. Since $d_{2}<u$ but $d_{2} \not \leq \wedge \varepsilon_{1}$, it follows that $u \wedge \varepsilon_{1}<u$, contradicting $u=\bigvee X$.

Case 3. $u=\varepsilon_{i_{1}} \wedge \varepsilon_{i_{2}} \wedge \ldots \wedge \varepsilon_{i_{n}}$, where $0 \leq i_{1}<i_{2}<\cdots<i_{n}<\omega$. Choose a $p>i_{n}, p<\omega$. Then $u \wedge \varepsilon_{p}$ is an upper bound for $X$. Since $a_{1} \wedge a_{2} \wedge \ldots \wedge a_{p}<u$ but $a_{1} \wedge a_{2} \wedge \ldots \wedge a_{p} \not \leq u \wedge \varepsilon_{p}$, it follows that $u \wedge \varepsilon_{p}<u$, contradicting $u=\bigvee X$.

Case 4. $u=\varepsilon_{i_{1}} \wedge \varepsilon_{i_{2}} \wedge \ldots \wedge \varepsilon_{i_{n-1}} \wedge \delta$, where $0 \leq i_{1}<i_{2}<\cdots<i_{n-1}<\omega$. Choose a $p>i_{n-1}, p<\omega$. Then $u \wedge \varepsilon_{p}$ is an upper bound for $X$. Since $a_{1} \wedge a_{2} \wedge \ldots \wedge a_{p} \wedge \delta<u$ but $a_{1} \wedge a_{2} \wedge \ldots \wedge a_{p} \wedge \delta \not \leq \wedge \varepsilon_{p}$, it follows that $u \wedge \varepsilon_{p}<u$, contradicting $u=\bigvee X$.

This completes the proof of the theorem.

Corollary . Let $E$ be a distributive algebraic lattice. Then $E[A]$ is complete (or algebraic) for every distributive algebraic lattice $A$ iff $E$ is finite.

Proof. Let $E$ be finite. We shall prove that $E[A]$ is an algebraic lattice. By Lemma $3, E[A]$ is isomorphic to $A[E]$; so we shall prove instead that $A[E]$ is an algebraic lattice. Let $P=J(E)$. Then $A[E]$ is isomorphic to $A^{P}$, and $A^{P}$ is a complete sublattice of $A^{n}$, where $n=|P|$. Since $A^{n}$ is an algebraic lattice, it follows that $A[E]$ is algebraic.

Conversely, if $E$ is an infinite distributive lattice, then $E$ has an infinite ascending or descending chain. Without loss of generality we can assume that $E$ has an infinite ascending chain. Let $A$ be a complete distributive algebraic lattice with an infinite complete-join independent antichain, for instance, the ideal lattice of the power set of an infinite set. Then by Theorem $5, E[A]$ is not complete.

Now we are ready to prove the main result:

Proof of Theorem 3. Let $L$ be a lattice, and let $D$ be a bounded distributive lattice.

Let $D$ be finite. Then the isomorphism (Con) follows from Theorem 1 .

Let Con $L$ be finite; again we have to verify the isomorphism (Con). Let $P=$ $J(\operatorname{Con} L)$. Now compute (observe that both $\operatorname{Comp} L$ and $\operatorname{Comp} D$ are lattices):

$$
\begin{array}{rlrl}
\operatorname{Con} L[D] \cong \operatorname{Id}((\operatorname{Comp} L)[\operatorname{Comp} D]) & \text { by Corollary } 1 \text { to Theorem } 4 \\
& \cong \operatorname{Id}((\operatorname{Comp} D)[\operatorname{Comp} L]) & \text { by Lemma } 3 \\
& \cong \operatorname{Id}(\operatorname{Comp} D)^{P} . &
\end{array}
$$

On the other hand, 


$$
\begin{aligned}
(\operatorname{Con} L)[\operatorname{Con} D] & \cong(\operatorname{Con} D)[\operatorname{Con} L] \text { by Lemma } 3 \\
& \cong(\operatorname{Con} D)^{P} \cong(\operatorname{Id} \operatorname{Comp} D)^{P} .
\end{aligned}
$$

So for Con $L$ finite, the isomorphism (Con) is equivalent to the isomorphism

$$
\operatorname{Id}(\operatorname{Comp} D)^{P} \cong(\operatorname{Id} \operatorname{Comp} D)^{P},
$$

which follows from Lemma 2 .

Conversely, let us assume that both $D$ and Con $L$ are infinite. Then $D$ is distributive, so it contains an infinite ascending or descending chain, $x_{1}, x_{2}, \ldots$.

We set $A=\operatorname{Con} D$ and $E=\operatorname{Con} L$. Then $E$ is an infinite distributive lattice, so it contains an infinite ascending or descending chain.

Case 1. $E$ contains an infinite ascending chain. $A$ is a complete distributive algebraic lattice with an infinite complete-join independent antichain, namely, $\left\{\Theta\left(x_{i}, x_{i+1}\right) \mid i=1,2, \ldots\right\}$. So Theorem 5 applies, and $E[A]$ is not complete. Thus the required isomorphism (Con) cannot hold since the left side is complete, while the right side is not.

Case 2. E contains an infinite descending chain. In this case, we choose in $A$ an infinite complete-meet independent antichain:

$$
\left\{\bigvee\left(\Theta\left(x_{j}, x_{j+1}\right) \mid j \neq i\right) \mid i=1,2, \ldots\right\}
$$

and apply the dual of Theorem 5 .

\section{REFERENCES}

[1] B. A. Davey, D. Duffus, R. W. Quackenbush, and I. Rival, Exponents of finite simple lattices, J. London Math. Soc. 17 (1978), pp. 203-211.

[2] D. Duffus, B. Jónsson, and I. Rival, Stucture results for function lattices, Canad. J. Math. 33 (1978), pp. 392-400.

[3] G. Grätzer, Universal Algebra, Second Edition, Springer-Verlag, New York-Heidelberg-Berlin, 1979.

[4] G. Grätzer and H. Lakser, Chain conditions in the distributive free product of lattices, Trans. Amer. Math. Soc. 144 (1969), pp. 301-312.

[5] G. Grätzer, H. Lakser, and R. W. Quackenbush, The structure of tensor products of semilattices with zero, Trans. Amer. Math. Soc. 267 (1981), pp. 503-515.

[6] A. Mitschke und R. Wille, Freie modulare Verbände $F M\left(D M_{3}\right)$, Proc. Univ. Houston Lattice Theory Conf., Houston, 1973, pp. 383-396.

[7] R. W. Quackenbush, Free products of bounded distributive lattices, Algebra Universalis 2 (1972), pp. 393-394.

[8] E. T. Schmidt, Remark on generalized function lattices, Acta Math. Hungar. 34 (1979), pp. 337-339.

[9] M. Tischendorf, The representation problem for algebraic distributive lattices, $\mathrm{Ph}$. D. Thesis, Darmstadt, 1992.

Department of Mathematics, University of Manitoba, Winnipeg, Man. R3T 2N2, CANADA

E-mail address: George_Gratzer@umanitoba.ca

Department of Mathematics, Transport Engineering Faculty, Technical University OF Budapest, MÜEgYetem RKP. 9, 1111 Budapest, Hungary

E-mail address: h1175sch@ella.hu 